\title{
Testing a Simple Model of the Unipolar Electrogram in the Intact Human Heart and Examples of Applications
}

\author{
Michele Orini ${ }^{1,2}$, Peter Taggart ${ }^{1}$, Pier D Lambiase ${ }^{1,2}$ \\ ${ }^{1}$ University College London, London, United Kingdom \\ ${ }^{2}$ Barts Heart Centre, St Bartholomew's Hospital, London, United Kingdom
}

\begin{abstract}
The unipolar electrogram (UEG) is widely used in electrophysiological research and in the cathlab. We aimed to test a previously proposed simple model of the UEG against in-vivo human data and to use the model to investigate: (A) Accuracy of repolarization measurements; $(B)$ Factors affecting UEG substrate mapping and $(C)$ Interactions between APD and UEG T-wave alternans. UEGs were recorded in 10 patients using a multi-electrode sock. Local action potentials showing same activation and repolarization sequence as measured in-vivo were generated using analytical functions. Local UEGs were simulated as the difference between the local action potential and a position-independent component representing remote activity. Morphological correlation between recorded and simulated $U E G$ was $c c=0.92(0.79-0.97)$ (median Q1$Q 3, N=1,756)$. Simulation studies showed: (A) Caution should be used when analyzing biphasic T-waves and Twaves associated with either very early or late repolarization. (B) Substrate mapping using UEG amplitude depends on the activation sequence and its total duration. (C) UEG TWA is not a specific surrogate for local APD alternans as it can be observed in sites without APD alternans due to variations in the remote component. In conclusion, the simple model provides a framework to improve the understanding and clinical utility of the UEG.
\end{abstract}

\section{Introduction}

The unipolar electrogram (UEG) allows simultaneous multi-site assessment of cardiac excitation and recovery and is widely used in electrophysiological research and in the cathlab. A simple models was previously proposed to explain its morphology in terms of local and remote activity [1]. The aims of this study were: (1) To test the model against in-vivo human data. (2) To use the model to investigate: (A) Accuracy of standard repolarization measurements; (B) Factors affecting unipolar substrate mapping and $(\mathrm{C})$ Interactions between APD and UEG T-wave alternans.

\section{Methods}

A ventricular mesh was created to reproduce the geometry of the multi-electrode sock used for in-vivo human recordings [2-4]. Local action potentials (APs) were generated at each node using the following analytical functions:

$$
\begin{aligned}
V_{i}(t) & =A \frac{1}{1+\exp \left(-\beta_{A T}\left(t-\tau_{A T, i}\right)\right)} \\
& \left(1-\frac{1}{1+\exp \left(-\beta_{R T}\left(t-\tau_{R T, i}\right)\right)}\right)-V_{0}
\end{aligned}
$$

where $\beta_{A T}$ and $\beta_{R T}$ are constant that determine the steepness of the activation upslope and repolarization downslope, respectively, of all APs; $A=10$ and $V_{0}=-90$ $\mathrm{mV}$ are the amplitude and the resting potential of $V_{i}(t)$; $\tau_{A T, i}$ and $\tau_{R T, i}$ are the AT and RT at cardiac site $x_{i}$, defined as $\tau_{A T, i}=\arg \max \left(d V_{i}(t) / d t\right)$ and $\tau_{R T, i}=$ $\arg \min \left(d V_{i}(t) / d t\right)$. According to a simple model prposed by Potse et al [1], the corresponding local UEG is:

$$
U_{i}(t)=C\left(V_{\mathrm{R}}(t)-V_{i}(t)\right)
$$

where $C=0.25$ is a scaling factor that takes into account the difference between intra- and extracellular conductances [1], and $V_{\mathrm{R}}(t)$ is a position-independent potential that represents far field activities, defined as the arithmetic mean of all $V_{i}(t)$.

\subsection{Analysis and Model Implementation}

Whole-heart epicardial contact mapping was performed in 10 patients during open-heart cardiac surgery using a multi-electrode sock enabling the acquisition of 240 UEGs [3]. S1 drive trains of 30-50 beats were delivered from one of the epicardial electrodes at cycle lengths equal to $500 \mathrm{~ms}$. UEGs were recorded at a sampling rate of $1 \mathrm{KHz}$ within $0.05500 \mathrm{~Hz}$, and referenced to the rib retractor. Signal averaging was conducted to reduce background noise. 
Data analysis was conducted with bespoke software as in other studies [5-7]. AT and RT were measured as the intervals between the pacing stimulus and the time of the minimum of the first derivative during the QRS complex and the maximum of the first derivative of the T-wave independently of its polarity, respectively. The local AT and RT measured in-vivo were used to generate corresponding local APs showing the same AT and RT, i.e. by modifying $\tau_{A T, i}$ and $\tau_{R T, i}$. The pair of position-independent parameters $\left\{\beta_{A T}, \beta_{R T}\right\}$ was chosen as that providing the highest median correlation coefficient (best morphological matching) between real and simulated UEGs among all combinations of $\beta_{A T}=\{0.2,0.4,0.6\} \mathrm{ms}^{-1}$ and $\beta_{R T}=$ $\{0.025,0.035,0.045,0.055\} \mathrm{ms}^{-1}$, while $\alpha=0.25$ in all configurations.

\section{Model Validation}

Based on signal quality and signal to noise ratio, 1756 UEGs were included in the analysis. Pooling together all data, correlation between recorded and simulated UEGs was equal to $c c=0.92(0.79-0.97)($ median Q1-Q3). Patient by patient analysis confirmed high morphological similarity between recorded and simulated unipolar electrograms, with median correlation coefficient equal to $0.89 \pm 0.061$.

\section{Insights from the model}

\subsection{Repolarization time measurement}

In the proposed model, the local RT corresponds to the maximum downslope of the action potential during repolarization. In the UEG, this corresponds to the maximum up-slope of the T-wave of the UEGs, except when the rate of variation of the remote component is not negligible with respect to the rate of variation of the local component, in which case a small bias is introduced. Therefore, the results of this study add further evidence to previous studies in support of the validity of the maximum upslope of the T-wave as a marker of the local RT [8]. However, this simple model also indicates some potential limitations of this method. In particular, sites with RT closer to the average RT may be more sensitive to noise and prone to RT estimation error, because as demonstrated in Fig. 1 they show smaller and biphasic T-waves. On the other hand, according to the presented simple model, while the effect of the rate of variation of the remote component is negligible for most of the sites, it may be sufficient to introduce a small bias in the RT of sites that repolarize either early (Fig. 1, left) or late (Fig. 1, right). This is due to the fact that during the beginning and the end of the repolarization process, the first derivative of the remote component is not constant and has a greater effect on the derivative of the local component (Fig. 1).

\subsection{Substrate mapping}

The amplitude of the unipolar signal can provide useful complementary information during substrate mapping for catheter ablation, in particular for endocardial assessment of transmural or epicardial scar. However, its amplitude depends on the activation sequence and varies substantially between sinus rhythm and ventricular pacing. The proposed model provides a simple explanation for both these characteristics. As the amplitude of the UEG is proportional to the difference between the local AP and the remote component, and since the remote component is equal to the average of all APs, the amplitude of the QRS increases with the difference between the local and the mean ATs. Therefore, the same site can exhibit different QRS amplitudes depending on the activation sequence, the local AT and the total dispersion of activation. This is confirmed by the results of a simulation study designed to investigate the interaction between UEG amplitude, AT sequence and total dispersion of activation. Three situations were simulated to reproduce AT sequences during sinus rhythm and ventricular pacing with total AT equal to either $110 \mathrm{~ms}$ or $150 \mathrm{~ms}$, respectively. In each case, the (absolute) QRS amplitude was related to the local AT through a U-shape association (Fig. 2A), since UEGs always show prominent QS and R waves at earliest and latest sites of activation, respectively, and a smaller QRS complex at sites whose AT is close to the average AT. During sinus rhythm, the QRS amplitude was smaller than during ventricular pacing because the activation is fast and spread out almost simultaneously in large portions of the ventricular tissue (Fig. 2B). During ventricular pacing, the beat associated with a greater AT dispersion (slower conduction) had larger QRS amplitudes (Fig. 2B).

\subsection{Repolarization alternans}

Repolarization alternans is a periodic variation of the APD occurring every other beat and is associated with increased risk of ventricular arrhythmia. The intra-cardiac UEGs allows measuring repolarization alternans and its spatial distribution by tracking changes in the local ARI. However, the interaction between repolarization alternans and TWA in the intra-cardiac UEG is unknown. According to the proposed model, local UEG-TWA is not necessarily due to local APD alternans and theUEG-TWA magnitude is not necessarily related to the magnitude of APD alternans. This can be explained by the fact that the morphology of the T-wave of the UEG depends on both local and remote activity. Figure 3 shows the results of a simulation study that assessed the causal link between the spatial 
distribution of APD alternans and UEG TWA. In a 500 nodes mesh, APD alternans with a magnitude of $5 \mathrm{~ms}$ was randomly distributed in an increasing number of cardiac sites, covering form $20 \%$ to $100 \%$ of the entire tissue. At sites showing both APD and UEG-TWA, the magnitude of TWA was inversely related to the proportion of sites showing APD alternans (Fig. 3A), meaning that inhomogeneity in the distribution of APD alternans tends to increase TWA. This can be explained by the fact that the larger the number of sites showing APD alternans, the greater the morphological beat-to-beat change in the remote component that compensate for the change in the local component due to local APD alternans. TWA was also present in sites not exhibiting APD alternans. At these sites, the magnitude of TWA was positively associated with the proportion of sites showing APD alternans (Fig. 3B). In this case, TWA is due to the beat-to-beat variations in the remote component and its magnitude is therefore the same for all sites not exhibiting APD alternans. According to the model, TWA due to local APD alternans can be distinguished from TWA secondary to remote activity since the former tends to have higher magnitudes (Fig. 3A-B) and it mainly appears during the up-slope of the T-wave (Fig. 3C), which is related to local repolarization, while the latter is equally distributed between the up-slope and the down-slope of the T-wave (Fig. 3D), which is related to remote activity.

\section{Discussion}

Good agreement was found between the morphology of simulated and recorded UEGs, supporting the validity of the simple model proposed by Potse et al [1]. The results are in agreement with [4]. The proposed model was applied to investigate the use of the UEGs in three applications with both physiological and clinical implications. The results of the studies support the use of the Wyatt method for RT assessment and suggest that caution should be used when analysing biphasic T-waves, which being smaller can be more affected by noise, and T-waves associated with either very early or late repolarization, as measurements may be biased by non-negligible effects of the remote component. The results of the second application suggest that when using QRS amplitude for supporting substrate mapping, one should take into consideration that the amplitude of the UEG depends on both local AT, i.e. the type of wave-front propagation, and total AT dispersion, and therefore different results could be obtained during sinus rhythm and ventricular pacing, with the latter also being affected by the pacing site and type of propagation. The results of the third application suggest that local APD alternans induces TWA in the UEG, which mainly manifests as a change in the up-slope of the T-wave (local component) of the UEG. However, UEG TWA is not a spe-
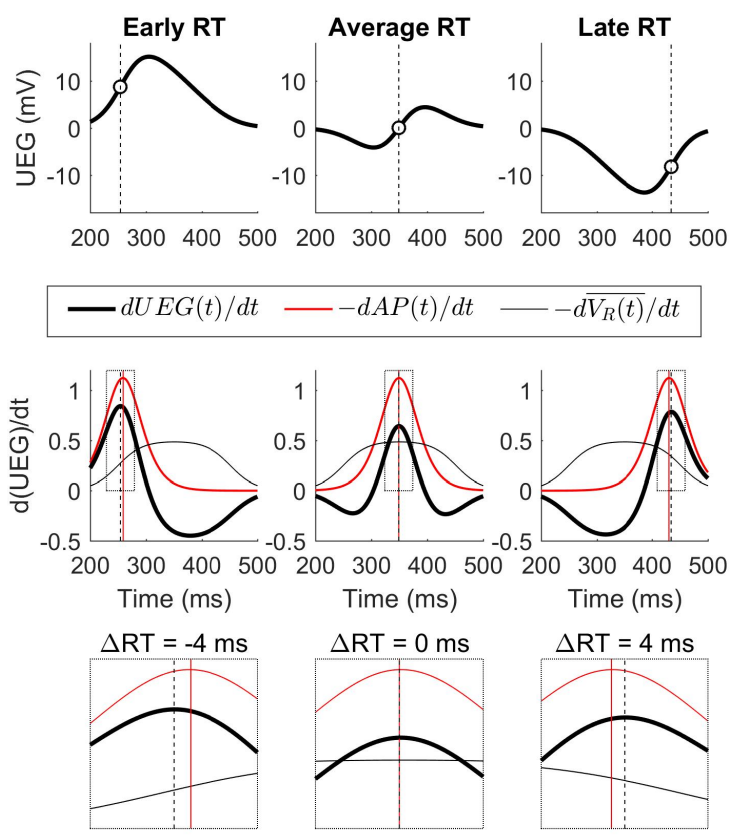

Figure 1. Simulation study showing the validity and possible limitation of the Wyatt method. Top panels: UEG associated with very early (left), median (middle) and very late (right) repolarization time (RT). Middle panels: First derivative of the local UEG (bold solid line), of the local AP (red line) and of the remote component (thin solid line).The black dashed and red vertical lines represent the maximum of the first derivative of the local UEG and AP, respectively, indicating that RT from the local AP and UEG are the same for almost the entire duration of the repolarization process and slightly differ for very early and late RT (left and right panels, respectively), when the derivative of the remote component is not flat. Bottom: Zoom from the middle panel.

cific marker of local APD alternans, as it can be observed in sites without APD alternans due to variations in the remote component that affect both the up-slope and downslope of the T-wave.

\section{References}

[1] Potse M, Vinet A, Opthof T, Coronel R. Validation of a simple model for the morphology of the $\mathrm{T}$ wave in unipolar electrograms. American journal of physiology Heart and circulatory physiology 2009;297(2):H792-H801. ISSN 0363-6135.

[2] Taggart P, Orini M, Hanson B, Hayward M, Clayton R, Dobrzynski H, Yanni J, Boyett M, Lambiase P. Developing a novel comprehensive framework for the investigation of cellular and whole heart electrophysiology in the in situ human heart: Historical perspectives, current progress and future prospects. Progress in Biophysics and Molecular Biology 2014;115(2-3). ISSN 00796107. 


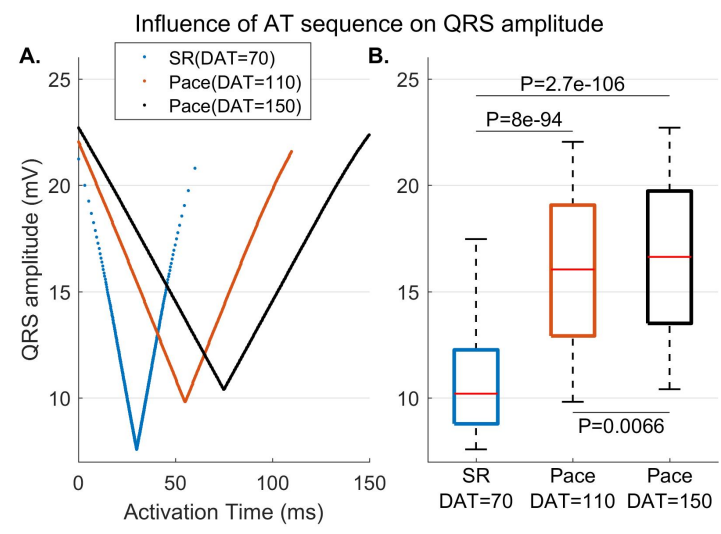

Figure 2. Simulation study investigating the factors determining the UEG amplitude. Three activation sequences were simulated in a 500 node mesh. Total dispersion of activation was DAT $=70 \mathrm{~ms}$ (sinus rhythm, SR), DAT $=110$ $\mathrm{ms}$ (pacing) and DAT $=150 \mathrm{~ms}$ (pacing). A: Association between the UEG amplitude (the absolute maximum within the QRS complex) and local AT. B: Boxplot representing the distribution of UEG amplitudes, indicating that on average slow activation is associated with higher amplitudes. P-values were computed with the Wilcoxon rank-sum test.

[3] Orini M, Taggart P, Srinivasan N, Hayward M, Lambiase $\mathrm{PD}$. Interactions between activation and repolarization restitution properties in the intact human heart: In-vivo wholeheart data and mathematical description. PLoS ONE 2016; 11(9):e0161765.

[4] Orini M, Taggart P, Lambiase PD. In vivo human sockmapping validation of a simple model that explains unipolar electrogram morphology in relation to conductionrepolarization dynamics. Journal of Cardiovascular Electrophysiology jul 2018;29(7):990-997. ISSN 15408167.

[5] Orini M, Citi L, Hanson BMB, Taggart P, Lambiase PDP. Characterization of the causal interactions between depolarization and repolarization temporal changes in unipolar electrograms. In Computers in Cardiology, volume 40. IEEE, 2013; 719-722.

[6] Orini M, Taggart P, Hayward M, Lambiase PD. Spatiotemporal characterization of the transition from sinus rhythm to ventricular fibrillation during an acute ischemic event in the intact human heart by whole-heart sock-mapping. HeartRhythm Case Reports 2017;3(5):253-257.

[7] Orini M, Taggart P, Hayward M, Lambiase P. On how 2/1 conduction block can induce T-wave alternans in the unipolar intracavitary electrogram: Modelling in-vivo human recordings from an ischemic heart. In Proceedings of the Annual International Conference of the IEEE Engineering in Medicine and Biology Society, EMBS. Institute of Electrical and Electronics Engineers Inc., 2015; 5676-5679.

[8] Coronel R, de Bakker JMT, Wilms-Schopman FJG, Opthof T, Linnenbank AC, Belterman CN, Janse MJ. Monophasic action potentials and activation recovery intervals as measures of ventricular action potential duration: Experimental
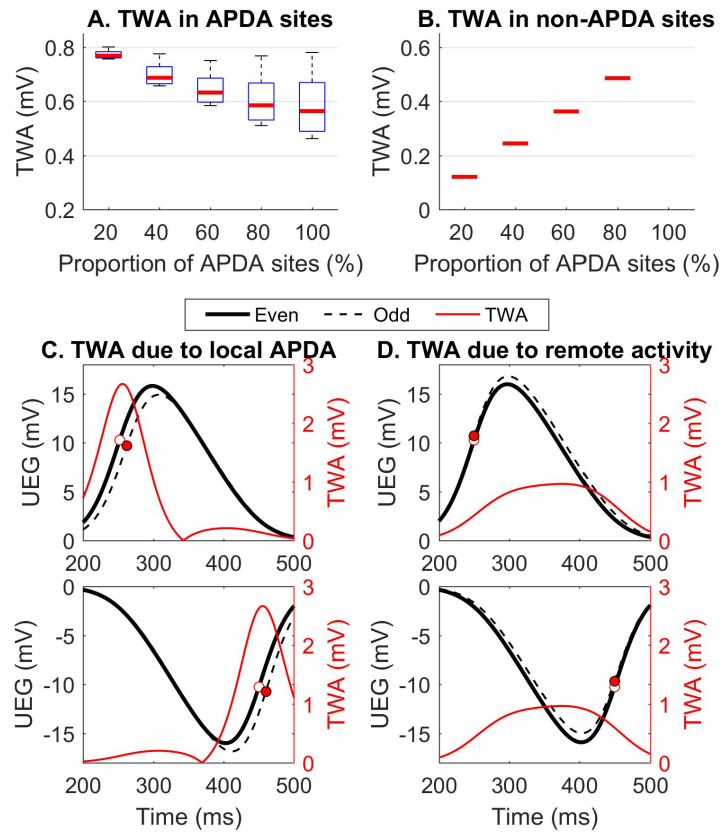

Figure 3. Simulation study investigating the interaction between APD-alternans (APDA) and T-wave alternans (TWA) in the UEG. A: In cardiac sites with APDA, the magnitude of TWA decreases with the proportion of APDA sites, showing that spatial inhomogeneity of APDA increases TWA. B: Result from cardiac sites without APDA, showing that TWA can be also detected in sites without APDA. In this case, TWA is due to the variation in the remote component and its magnitude is the same for all sites not exhibiting APDA. Bottom: Examples for both positive and negative T-waves showing that in sites with APDA, TWA (red line) mainly appears during the upslope of the T-wave as it is associated with local RT variation (C), whereas in sites without APDA, TWA is smaller and distributed over both the up-slope and down-slope of the T-wave as it is due to changes in the remote component (D).

evidence to resolve some controversies. Heart Rhythm 2006; 3(9).

Address for correspondence:

Name: Michele Orini

Full postal address: University College London, Gower Street, London, UK.

E-mail address: m.orini@ucl.ac.uk 